\title{
The Nature of Fundamental Consciousness
}

Karl Sipfle

Independently, at NASA GSFC

ksipfle@umich.edu

Nov. 17, 2020 


\begin{abstract}
In humanity's quest to understand consciousness, much work has been done at the biological and neural levels [1], some also in understanding cognitive higher consciousness [2], and a bit on the contribution and importance of value [3]. But as we have seen, all of this has not led to an understanding of fundamental consciousness. We know a good deal about how the networks of neurons in our heads calculate and process information, shedding light on how we think. This does not tell us how we feel. A mind is thinking plus, especially, feeling. In this article we consider basic mechanisms, the missing explanatory glue from the physical world to value and consciousness. We thus lay out the Sentonic Theory of Fundamental Consciousness (STFC), and end with the topic of sites in the brain where these phenomena may take place.
\end{abstract}

\title{
Keywords
}

Consciousness, physics, evolution, feeling, qualia, mind, cognitive, affective

\section{Consciousness as a Natural Phenomenon}

Let us note that consciousness is part of Nature. We speak of the "physical world," tacitly excluding from any mention in our physics models such things as pain and pleasure. This is incomplete physics, and certainly can never be a theory of everything that exists.

Pain and pleasure are items that must be added to the list of phenomena that physics- the description of Nature- is to describe and characterize. Furthermore, they must be added, if provisionally, to the list of fundamental phenomena, for we are unable to derive them from anything in our standard models, which stand in isolation from them.

What has always turned out to underlie all phenomena? Particles and fields. Until there is proof to the contrary, the simplest assumption is that these phenomena are a dance of their own particle(s) and field(s).

\section{The Root of Value}

To operate, our minds utilize both information processing and value, the latter ultimately determining the course of the former. Though now abstracted and cooled in some parts of the cortex, value in animal minds originates from pain and pleasure. 


\section{Characteristics of Consciousness, Characteristics of Physics}

In pondering consciousness over the ages, humanity found itself in a circle: who is observing that which is what observes? The answer is that elementary consciousness must be free-standing. At the lowest level, you do not feel something, the feeling simply is.

Because we can construct the entire Standard Model without ever mentioning consciousness, we see that elementary consciousness is fundamental not just in the scope of animals but in the scope of the universe. It is an ingredient of reality that is missing from our physics.

We have been here before. A large basket of phenomena was ultimately traced to the electromagnetic forces. So, too, other discovered phenomena very apart from the compiled theory, were discovered to arise from the strong and weak nuclear forces. Now, everything that happens in the so-called "physical" world is known to be a result of these forces plus gravity.

In our Standard Model of physics, each fundamental occurrence possible is described by a quantum force field, at least one corresponding particle mediating that force, known as a boson, and a charge (a quality) that particles may possess ("carry") that make them participants in that force [4].

\section{The Standard Way of Extending the Standard}

When we discover something new that appears to be the same everywhere, such as, possibly, dark energy, we may postulate a new characteristic of "empty" space. But where there is change across space, the simplest and eventually proven solution for odd phenomena has always been new particles. In the less odd cases these found particles just have a different combination or different strengths of known charges. For the oddest phenomena, charges new to us were at work, and ultimately discovered.

One new force field, consistent with the Standard Model, was recently proposed and then confirmed (the Higgs field), and other fields have been hypothesized to explain inflation and dark energy.

A leading explanation for dark matter rests on a new, undiscovered particle, which is thought to possibly interact with an as-yet undiscovered force field (and thought to produce neutrinos when annihilated).

\section{The Consciousness Field}

Hence, we hypothesize a new field, the consciousness field. As with all fields, it corresponds to at least one new boson (force particle) which we call the "senton" (from sentience), and acts on particles that carry the consciousness ("sentonic") charge.

As a boson it will have an integer spin and obey Bose-Einstein statistics. There is no requirement to introduce any new matter particles (fermions), just the new charge that known matter particles may carry. 
A consciousness (i.e., sentonic) field is a vector field and not a scalar field like the Higgs field, because it trails off with distance; I don't feel what you do.

Many specific candidates present themselves as the base sentonic actions in our world, the events that generate consciousness phenomena. The creation, movement, rotation, spin flip, spin alignment, absorption, and/or decay of a sentonic-charge-carrying particle or a senton is when fundamental consciousness happens and what it is.

Molecular combining, disassociating, and reconfiguring actions are quantum state changes that could include sentons as well as photons, as could atomic orbital changes. This throwing off and absorbing of sentons instead of just photons as they change quantum states suggests that a quantum wave function collapse would then correspond to a consciousness event. It is particularly interesting that the exact meaning of the collapse of the wave function, and the exact nature of consciousness, are both unanswered questions.

Photoreceptor molecules in the retina, which is an outgrowth of cortex, change shape upon absorbing a photon, which causes the cell to emit spikes. Sentioreceptor molecules in other cortex may do the same thing.

The crucial quantum mechanisms for consciousness might therefore include:

- Photons changing the spin of sentonically-charged particles as they do with electrically charged particles.

- Sentons changing the spin of electrically charged particles.

- A senton decaying into a photon (though we predict later that it is probably stable).

- Sentons propagating for long distances as consciousness radiation (and participating in quantum teleportation).

- The collapse of probability waves, which may also exist for consciousness itself.

- A possible link with dark energy which, like consciousness, must be fundamental and yet not directly detectable. If they are the same, most of the energy of the universe is consciousness.

- A parallel of gravity waves, propagating across the hidden dimensions of String Theory

Both more theoretical analysis and more experimental input are needed to winnow the field of possible mechanisms. Meanwhile, the above collection as a whole provides us with a class of theories- a metatheory-from which the specific case may later emerge. We call this metatheory the Sentonic Theory of Fundamental Consciousness (STFC). It starts with the realization that fundamental consciousness requires a new field, and proceeds through the resulting implications. 


\section{Base Consciousness and Qualia}

Noting that consciousness is real, we have been driven to our conclusions thus far by two entirely different paths that converge. On the one hand, philosophical study of the consciousness problem leads us to the conclusion that consciousness must be something that happens on its own and is not an experience by another thing of the thing in question. When it happens, consciousness simply is. On the other hand, an examination of our current detailed physical model of the universe does not mention consciousness. Therefore, this model of reality is incomplete and must be extended. We extend it in the way that is simplest and most consistent with the existing (and very successful) model, which is by a new, fundamental field.

Once this base feeling- negative and positive- is in place, more complicated things that people also call consciousness may be built therefrom (the "mental stack"). So, pain and pleasure are the base consciousness phenomena, but we believe that other familiar qualia are not fundamental conscious experiences. If they were, there would have to be a fundamental force for "redness," and one for each in a tremendous plethora of other experiences in humans (and probably many animals and extraterrestrials, too). Our experience in physics to date tells us this is unlikely. Most qualia are elaborations, incorporating information, at a higher level of discourse. Any experience, large or small, aside from pure pain or pleasure, will have a different what-it-is-like, caused by a particular live mosaic of interacting points of consciousness and matter. This is a very important point. The assumption that typically mentioned qualia mined from introspection and the smallest consciousness bits of the universe are the same has led many astray.

\section{Building the Mental Stack}

Above Fundamental (or "base") consciousness, which is the scope of this article, we have, in animals, "Low consciousness." This is what is commonly called sentience, provided by virtue of a substantial enough brain. It is what happens in the simplest organism we are willing to call sentient and involves intake of information. It is the beginning of Mind.

Built on this we have "High consciousness," the large realm of everything up through cetaceans and primates. Thinking appears here. It is everything our own minds have except the precise and organizing capabilities of language.

Built on this we have Human consciousness, language-powered and capable of formal thought and advanced problem-solving and self-reflection. It is assumed the reader is familiar with this.

Built on that will be Suprahuman consciousness, the nature of which is more speculative, and will likely result from integration with machines, genetic modification, or both, and possibly be present in freestanding machines of ours (of a new kind). It has surely already appeared with some extraterrestrials. 


\section{Brain and Consciousness}

The brain is made of matter particles, most of which are electrically charged, and many of which are in motion. The brain has many electrical activities going on with special and unusual patterns, in close molecular quarters of special shapes.

Higher up, the brain is made of neurons, the only part making it strikingly different from the other organs. There is one thing that makes neurons strikingly different from other cells: the production and transmission of neural spikes. As these spikes are reduced, such as by drugs, consciousness indeed fades.

The generation and transmission of neural spikes fundamentally involves the motion of electrically charged matter in the form of elemental ions.

Neurons have receptors for chemicals, but so do all other cells. All cells have ion channels in their outer membranes, but only neurons and the recipient muscle cells have voltage-gated ion channels for transporting potassium, calcium, and sodium. These generate and propagate the spikes, stimulated or suppressed by neurotransmitter molecules crossing the synaptic gaps between neurons and binding to receptors, which turn opens and/or closes ion channels.

The movement of ions is a key phenomenon in the brain. Since the charged carriers are ions, their nuclei bring the strong and weak nuclear forces, but the mere movement of particles with these nuclear charges is commonplace in Nature, not special to the brain.

Electric charge, however, is extremely significant in the operation of the brain. In a transmitted spike, matter does not appear to move in any way that is particularly notable for matter, but the voltage waves do, as they are relayed from spot to spot along the matter. That leads us to believe that the connection in physics between the brain and consciousness boils down to the connection between movement of electric charges (which happen to be on ions) and consciousness, at least at the beginning of the process.

The simplest expectation is of a direct link without conversions in the middle. This would mean an electrically charged matter particle has the ability to move, or to be, a sentonically-charged particle. And that implies there is either an innate link between the two forces, electromagnetic and sentonic, or a particle exists carrying both charges, which serves as the link. Thus far in our argument, this makes candidates of all of these: electron, quark, photon, and senton. A new kind of interaction between one or more of these four kinds of particles, or possibly simply the movement of one of them, corresponds directly to- is- the phenomena we experience as pain and pleasure, set in motion in our world by electromagnetic action.

Next, we note this: In order for consciousness to have a useful effect, it must in turn affect the behavior of neurons. In other words, interaction must run in both directions (rather like the link between electricity and magnetism), from electromagnetic to sentonic and from sentonic to electromagnetic. 


\section{Fusion in the Field}

We have seen earlier the expectation of consciousness as a separate, fundamental action both from a physics and a philosophy starting point. The field called for by physics also harmoniously explains something very important seen in the philosophy and experience of mind.

Emotion adds and fuses. Both traits are important, defining characteristics for us. As you become more and more happy or distraught, undoubtedly caused by more intense firing of neurons and more of them doing so, the feeling becomes more intense, and it is singular. What you do not feel (or more properly, what is not happening in you), are more, separated independent feelings. The physics-level events, these little freestanding dots of fire, are connected. The feeling is a large, fused whole [5].

This is exactly what happens in a physical field, be it sentonic or electromagnetism or gravity. Each point in space experiences the sum of force from all charges around it, and a continuous blob is the result. That is the feeling within you (or within each of a few separate places in your brain). And that is how Fundamental consciousness becomes the emotional part of higher consciousness.

This also means that each neuron that affects the blob thereby affects all other neurons in contact with it, providing a bath of general how-things-are-going, a mechanism of great use to evolution in developing a complex, aware, and adaptive brain.

\section{Properties of the Senton}

To eventually suggest experimentally detectable properties of the particles and field we propose, we need to take the argument further. The senton is the boson that mediates the interaction between particles with sentonic charge. In this section we make plausible, qualitative forecasts of its properties, congruent with existing laws.

Present knowledge drives us to start from the following likelihoods:

- Known bosons with mass have extremely short range, and sentonic action needs range to fuse events on brain scales; so the senton has no rest mass.

- All zero rest mass particles are stable; so the senton is stable.

- Most bosons have no electric charge, and none of the long-range ones do, and no boson without rest mass has electric charge; so the senton has no electric charge.

- Most of the boson classes and all of the long-distance bosons have no color charge; so the senton is not color-charged.

- While the weak force $\mathrm{W}$ bosons have weak isospin (but no weak isocharge), they also have both mass and electric charge; so the senton is not weak-charged.

- None of the long-distance bosons have the charge on which they operate; so the senton is not sentonically-charged.

These senton qualities make a senton indistinguishable from a photon. This means either 
1. Sentons work in different dimension(s); or

2. The photon is the force carrier for fundamental consciousness.

Or else some of our provisional deductions need to be altered, e.g.:

3. The sentons do carry sentonic charge and react with each other, whereas the photon does not carry sentonic charge. This case is interesting because it would further promote a fusion of feeling.

4. The sentons do not carry sentonic charge and react with each other, whereas the photon does carry sentonic charge.

5. The senton does carry electric charge. This seems unlikely since we've never detected it.

6. The senton does carry color charge.

7. The senton does carry weak charges.

8. The senton does have a little rest mass.

We cite $1,2,3$, and 4 as the most likely possibilities: the senton and photon differ in that they operate across different dimension sets, or in that one but not the other carries the sentonic charge, or they are, in fact, the same particle.

So far, all known charges are not just mediated by bosons but also carried by some boson. We anticipate at least one boson carrying the sentonic charge. The cleanest situation is if this is the photon or the senton. So far, the photon is the only particle not known to carry any kind of charge (except for the Higgs boson, which is the only known scalar boson).

If sentons also carry the sentonic charge, they interact with each other. Bits of fundamental consciousness affecting each other is necessary for a successful physical theory of consciousness, given our personal knowledge of pain (or pleasure) adding into one experience. We have theorized that this happens because of the sentonic force field in which matter particles containing sentonic charge interact. Sentons themselves carrying sentonic charge would be another route for bits of consciousness to fuse into an emergent whole (and perhaps enhance time persistence). It is possible that both things happen.

\section{Carriers of the Sentonic Charge}

Though not yet seen, it remains conceivable to have a force- a boson- that operates only on other bosons, with no matter particle carrying its charge. (It is also conceivable to have a boson that, once created from free energy, acts only on itself, but that would deprive us of the necessary feedback back into the neuron). This is a very interesting thought, because then we have a force with no "extended" objects requiring space (per [6]). As things stand, however, fermions as a group carry all known charges. This suggests that at least one fermion carries the sentonic charge.

Considering the four types of fermions (neutrinos, electrons, and the down and up quark), there does not immediately appear to be anything neutrino-ish about brain behavior. In contrast, fermions of the other three types all carry electric charge. Furthermore, all fermions have instances of weak 
hypercharge. The weak interaction is linked to electromagnetism as one half of the composite electroweak force. We have seen that sentonic action is somehow linked to the electromagnetic interaction. It is possible that all fermions (that is, all matter), can carry the sentonic charge as well.

Since the brain uses ions for electricity instead of electrons it is conceivable that the nucleons carry the sentonic charge, which means the quarks (or the gluons). Quarks are the only particles known to experience all known forces, that is, to have all known charges. We might ask if they experience the sentonic force and carry the sentonic charge as well. They also make themselves conspicuous as the only particles to carry fractional and asymmetric charges of, once again, electromagnetism.

Examining possible bosonic carriers, we remind that the gluon, $\mathrm{W}$, and $\mathrm{Z}$ are all very short-range, which appears to be a "deal-breaker" given our need for field effect on macroscopic (brain) scales. The Higgs field is scalar, providing insufficient variety of action across space. What we need is a force that operates effectively in the intermediate realm between very-short-range and everywhere, as does electromagnetism.

The remaining candidates to carry sentonic charge are the quark and electron fermions, the photon, and the senton itself.

The simplest cases of how electromagnetic behavior (as in the brain) could cause sentonic behavior (base consciousness), are where the same particle carries both electric charge and sentonic charge, which would also supply interaction in the reverse direction. As a criterion that would narrow the cast to quark and electron. A minimally more complicated alternative solution is that whatever boson mediates the electromagnetic force (the photon) also carries the sentonic charge (which is one of the scenarios we marked as likely in the last section), which expands the list to quark, electron, and maybe photon.

As all known forces are carried by a fermion and by a boson, we anticipate a minimal reality of at least the electron or quark, and the photon or senton, carrying the sentonic charge. Since it may be either a fermion or boson providing the electromagnetic to consciousness link, we do not know whether it is the photon or the senton that carries sentonic charge.

The three particles on the list that we do already know in Nature are all either electrically charged or mediate between things that are. The sentonic-carrying photon case also suggests the possibility that the senton carries electric charge, as a symmetric return path for the interactions. This would be a first case of a somehow electrically charged yet massless particle.

In summary, we propose that:

- The particles that carry the sentonic charge are most likely the electron, quark, photon, and/or senton.

- The predicted properties of the senton are spin 1, massless, stable, no color charge, no weak hypercharge or weak isospin, possibly sentonically-charged, and conceivably electrically charged.

\section{Sites of Action in the Brain- Macroscopic}

Let us now consider the most likely sites of action in the brain. 
This question is actually three: which neurons hold the sites of action, which regions of the brain contain these neurons, and where in the neurons is the site of action. For the first two questions- the macroscopic world of the cortical region and the cell- the evidence is stronger. For the third and most interesting- the sub-neural, molecular scale (where physics lives), we are further away from knowing.

Macroscopically and microscopically, some areas of the brain show no signs of consciousness. This suggests that certain neurons and not others have evolved conscious ("feeling") capability, directly causing pain or pleasure. This means the former have evolved nontrivial, nonchaotic access to the sentonic field.

It is medically known that human consciousness requires only

1. Cerebral cortex ("for awareness")

2. The Reticular Activating System of the brainstem ("for arousal")

The RAS projects to all cortex but mainly to prefrontal cortex.

If we look at limbic brain tissue as pain/pleasure-related, we find that it omits the stellate cells of cognitive tissue. This suggests that the feeling neurons ae pyramidal. Throughout the cortex pyramidal cells are the "workhorse" cells, the primary (and evolutionarily long-standing) units of the cortex, while the others provide regulation, lateral inhibition, relay, and pattern preprocessing to the deciding and publishing cells, the pyramidals. Closely associated with pyramidal cells, however, are chandelier neurons, whose axons always synapse exclusively on the axon initial segment of pyramidal cells. Therefore, chandelier cells may play a role in consciousness.

Pyramidals appear especially in cortical layers III and V so one or both of these is the most conscious layer(s).

Layers II and IV of neocortex contain many stellate interneurons and are evolutionary newer, added once consciousness was in place.

The pain (dysphoria) center can be found in the anterior cingulate cortex (Brodmann areas 24, 32, 33). This area is also involved in reward anticipation. It is unique in its abundance of spindle cell neurons, which are only found in very smart mammals. The spindle cells may not be themselves the site of consciousness, and do exist in other places, but their high density here may highlight the special importance of this area for very smart animals.

In pleasure (euphoria), orbitofrontal cortex and/or insular cortex are probably involved. The OFC is also involved in expectation of both reward and punishment in response to stimuli. 


\section{Sites of Action in the Brain- Molecular Scale}

Anesthetic experiments and pathology and injury studies indicate that it is not receptors and it is firing sequences that cause consciousness (and also that some neurons make consciousness and others do not). Most likely, spike generation or transmission is related to consciousness by a very short causal path.

Consciousness appears to occur in axons. The charge travel pattern of a neural spike is complex, unusual, and dimensionally precise. Here we have a toroid of deluges of ions across the axonal membrane, itself traveling along the considerable length of the axon.

This means the ion rush through these channels may be the site of consciousness.

While dendrites often taper, axons maintain a constant radius. The exact diameter varies by neuron type. It is typically about one micron. No neuron ever has more than one axon (though it may branch extensively).

The axon initial segment consists of a specialized complex of protein molecules.

Note that the physics of axonal spikes might be replicated by a constructible apparatus for experimental purposes. This would allow for more intense examination than is readily possible in a brain or even brain slice. Effects discovered could then be sought in real brain tissue.

Microtubules have been suspected in consciousness theories [7]. Much like axons but at a smaller scale, they are of even more constant and small radius and run the length of the axon and whole neuron.

They experience the toroidal voltage pulse running down the axon that possibly induces something in or on the microtubule. These precisely dimensioned microtubules might function as resonators of some kind. In our model they would produce resonances in the sentonic field, or of a causative agent. Subsequent spikes would regenerate a decaying resonance. Also worth noting is that many differing molecules bind to microtubules.

It is not clear what the exact mechanism is, but it is clear that there are several possible pathways for what is needed, which is ion control of sentonic events and vice versa. At a slightly higher level that is all we need, and we can await the elucidation of the details.

The sentonic field provides another means, requiring no direct cabling, for points in the brain to communicate with each other ("ephaptically").

While cycling (and resonance) of signaling in the recurrent connections in neural networks may provide a regenerative effect that fortifies consciousness, it is not the base mechanism (cycling is a base mechanism of short-term memory, however). 


\section{Evolution}

Pain and pleasure have been discovered and harvested by evolution. There are likely other worlds in which pain and pleasure don't exist to organisms, even complicated ones.

Pain and pleasure might not actually be the same force. And there might be animals that feel pain but no pleasure. Use of one may have come first in bio-evolution (and that would likely be pain).

Intensity of experience increases by way of more spots, and indirectly, by more related interconnections.

Harvesting fundamental consciousness by evolution would mean "crafting" the shapes and/or construction and constituents of neurons, and possibly sets of neurons.

We can recapitulate the evolutionary development sequence because the needs and difficulties are obvious, and because we have the animals around us as evidence.

First needed would be basic stimulus/response. This could be viewed as the degenerate case of the cognitive. Then would come totally dispassionate ability for slightly complicated perception and actions. In other words, calculating ability- pure, passive cognitive- would appear first, and exists in primitive animals.

Feeling provides for the first operant (reinforcement) learning opportunity, and certainly for more complicated organisms. It offers a broad whatever-you're-doing-is-bad (or good) signal for learning and it provides the second needed, broadly-distributed system- Value- which determines what action impulses are let through, the first system being Activation. Third is Attention, targeting and focusing the cognitive portions. (Even before well-developed learning appears, feeling allows for generalized realtime changes in decisions in creatures with some neural complexity, and serves Attention).

This progression into feeling began with the evolutionary outgrowth of the allocortex- the olfactory system and the hippocampus. The paleocortical piriform cortex, a.k.a. posterior orbitofrontal cortex (posterior OFC), is heavily correlated with the cingulate gyrus and the septal area, which is part of the basal forebrain. The OFC mediates the expectation of reward/punishment in response to stimuli. The paleocortical olfactory tubercle plays a role in transmitting positive signals to reward sensors.

Once all these lower mechanisms were in place, the neocortex burst forth into high growth. Warmer/Colder is a very powerful concept (complementing Recognition).

Comets or asteroids could have provided particles that contain sentonic charge. They could have come from anywhere or anyone.

\section{Applications and Technology}

Real artificial consciousness, entirely different from Al and not needed for truly-intelligent Al given simulated consciousness, is in principle doable. By this we mean creating truly conscious minds that are 
made by machines we build rather than biological brains. This means that their pain and pleasure, unlike simulated consciousness, would be real. They would be like us (and thus perhaps entitled to rights).

Simulated consciousness is like a simulated car or solar system. It would mimic the same behaviors, but would not be real, not even as a new form of real consciousness. That is because our minds are not just information flows, they have feelings. And feelings are real just as much as the steel of cars is real, they are just a different physical thing than moving steel.

A machine of today is not really conscious- not sentient- but can be effectively conscious-like, like a heat-seeking missile or drone. If we discover how to manipulate the sentonic field with an apparatus, then we may be able to make machines that are truly conscious. For example, the specific electrical pattern of the traveling wave in a neuron's axon is different from any other pattern encountered in nature. This pattern could be produced artificially, possibly resulting in not just simulated feeling but actual feeling. As advanced engineering, it would not even require us to understand exactly what those traveling waves are doing.

Artificial emotion could be achieved by creating engineered structures (perhaps nanostructures) similar to the critical elements of the brain. There is a potential practical application for this, in augmenting and continuing our brains, not with simulated unreal emotion, but with the real emotion that fundamentally and satisfyingly makes us us, rather than models of us.

How could an experimenter tell the difference? In a practical way. Method acting is the easier and more convincing technique because the feelings you in the audience are observing, with their myriad cues, are real. Trying to control every muscle movement in simulation like a Laurence Olivier is very difficult for even a real human to pull off convincingly. To our advanced brains, not-truly-feeling minds are likely to be detected or suspected, even though in principle they might fool an observer. Advanced and consistent emotional mindfulness exhibited by a myriad of behaviors and utterances with all their attendant details and choices will probably be detected correctly as real. This is an inversion of the Turing Test, where rather than purposely being remote from the new actor, one is purposely able to observe closely.

Providing the random numbers needed to support the mind algorithm by real random events in our universe (which ultimately come from quantum waveform collapses), rather than only pseudorandom calculated numbers, would complete the entry of this consciousness into a full joining with our real world. It would no longer be in any aspect a simulation, but rather, a constructed actual conscious mind living entirely in our world, out of the bottle. 
Note that genuine conscious feeling is not required for intelligence- a nonfeeling stand-in that did exactly the same function would do (and would basically appear to feel, even to itself), but it would not feel, it would at most think it feels. It wouldn't actually know "what it is like," which means to recall similar situations for the feelings they evoke. Its knowledge would be more like book knowledge. But genuine conscious feeling would not be necessary to display behavior equivalently "willful" to our own.

The first steps in trans-human fused consciousness would involve sensory, motor, cognitive processing, and perhaps simulated-consciousness implants. There would be new things to experience by the existing, re-purposed neurons, creating somewhat different new conscious experiences.

The next step would be joining true artificial consciousness with human consciousness. An advantage of this would be continuity as the original biological brain slowly dies off. The baton would be passed continuously from the original brain, to the joined and fused and co-experiencing brain, to the surviving portion of brain. This is fundamentally different from a clone or a download, which ensures a legacy but not a survival, except of data patterns. Before the advent of fused true consciousness, one can only pass on what amounts to a book. After its advent, one can instead change slowly, as in normal life, but the "soul," in the same patch of the sentonic field, is poured slowly from one flask to mix with another, and then slowly evaporate to a degree, together. Though the atoms in your own body are continually replaced, you don't question whether you are still you after years of this. At a minimum, your most intimate imaginable "partner" will live on, indefinitely, and probably with better memory- your memorythan we all have today.

Until such time, we might work to preserve our feeling neurons as long as possible and replace the ones with purely cognitive function, which might make preserving what matters of your brain and surviving as a person easier. How you feel and how you think are who you are, and the latter is just information processing. Learning somewhat different ways of adjusting our mechanical functions does not feel like a key change and loss of self, any more than does adopting an artificial limb.

On the journey, we may be able to feel entirely different forms of consciousness, dependent on what we are attached to. We have foreseen entirely new experience in a clear way: lust is a conscious experience a small child does not have or comprehend, yet the same person later does. New-sense implants will cause new things to know "what it is like."

\section{Summary}

The fact that it is difficult to understand does not make consciousness any less a natural phenomenon than electricity or nuclear fusion. The Standard Model incorporates everything we completely understand so far, and in the long run never fails to accommodate extension and revision to cover new discoveries. 
Being quite separate from the other phenomena, a new force and thus charge, field, and boson are scientifically called for. The new force interacts, by some chain of interactions, bidirectionally with the electromagnetic force, which is responsible for nearly all operations in the living brain.

Evolution has learned to harness this "new" force, which has existed latently for eons. Learning how to interact with it directly will allow the creation of feeling machines and the continuity of our developing selves for ages.

\section{Conflict of Interest Statement}

There is no conflict of interest for the work of any article written by me through the year 2020 .

\section{References}

[1] E. Kandel, Principles of Neural Science, New York: McGraw-Hill, 2013.

[2] E. T. Rolls, Cerebral Cortex: Principles of Operation, New York: Oxford University Press, 2016.

[3] G. Edelman, Bright Air, Brilliant Fire, New York: Basic Books, 1993.

[4] Kane, The Particle Garden, New York: Basic Books, 1995.

[5] K. Sipfle, The Nature of Consciousness, New York: Barnes and Noble Press, 2018.

[6] R. DesCartes, Principia philosophiae, Amsterdam:: apud Ludovicum Elzevirium, 1644.

[7] R. Penrose, The Emperor's New Mind, Oxford, UK: Oxford University Press, 1989. 